Classification

Physics Abstracts

$07.85-29.30-78.70 \mathrm{D}-81.70 \mathrm{C}$

\title{
A new quantification procedure for elemental mapping by $X$-ray (absorption) microscopy $\left({ }^{\star}\right)$
}

\author{
J. Cazaux \\ LASSI GRSM - BP. 347 Faculté des Sciences, 51062 REIMS Cedex, France
}

(Received 10 October, 1993; accepted 16 December, 1993)

\begin{abstract}
In this paper, the principles of a new procedure for analytical X-ray microscopy (AXRM) are given and they apply for any kind of microscopes based on the use of conventional X-ray sources or of synchrotron radiation. Over the differential approach, its advantages are to minimize the number of irradiations to be used and its ability to map elements (or species) even when the investigated photon energy range does not include the threshold energy of some components. An additional advantage is that it permits to distinguish between thickness contrast and chemical contrast of the initial images via the thickness mapping. The factors influencing the precision on the concentrations measurements are discussed and it is shown that the sensitivity may reach $10^{-5}$ at/at. for the detection of medium elements embedded in very light matrices for photons in the $5-20 \mathrm{keV}$ range. In fact, the measured intensities may be influenced by fluorescence effects and by the possible non-monochromaticity of the incident beams, mainly when classical X-ray sources are used (such as in X-ray projection microscopy) and correction procedures dealing with these effects are given. Some concepts developed here may also be adapted to conventional X-ray radiography and tomography.
\end{abstract}

\section{Introduction.}

Looking back at the progress of electron microscopy over the past twenty years, the most striking fact is clearly the spectacular development of analytical electron microscopy which has added a new dimension, that of elemental mapping, to the structural information usually obtained by conventional electron microscopy. The following cases are notable examples: electron energy loss spectroscopy (EELS) combined to a conventional Transmission Electron Microscope (TEM) or to a Scanning Transmission Electron Microscopy (STEM) [1, 2]; energy dispersive X-ray spectrometry (EDS) combined to a Scanning Electron Microscope (SEM) [3] or to a STEM [4]; Auger Spectroscopy combined to an ultra high vacuum SEM [5].

The benefit of adding elemental mapping to X-Ray Microscopy (X.R.M.) is obvious if the specific advantages of X-ray microscopy with respect to EM are considered: the ability to observe far thicker specimens even placed in selected environments (air, water). Of course, the principles of

$\left(^{\star}\right)$ Some aspects of this paper have been presented at XRM $4^{\text {th }}$, Chernogolovska, Russia, 20-24 September 1993. 
differential X-ray microscopy have been known for a long time [6-10]. They consist in obtaining two images of the specimen by using two different photon energies, $E_{1}$ and $E_{2}$, situated on the two sides of an absorption edge energy of the element to be visualized. This procedure is similar to that of EELS in its principles but it often requires the use of $2 n$ irradiations for the mapping of $n$ elements. More, only the local weights per unit area are obtained so that the final images are also sensitive to the local changes of the thickness of the specimen.

In fact, X-ray photon-matter interactions differ from electron-matter interactions because the main process is the absorption effect and it is not influenced by multiple scattering effects. The result is that the elemental information is present at any energy of a X-ray absorption spectrum and is not restricted to regions situated above threshold energies. Based on this remark, the goal of the present paper is to propose a new approach which only requires the use of $n$ radiations and which also permits to obtain thickness maps.

The new formalism to be applied for finding the atomic concentrations of the $n$ elements composing a specimen as a function of the $n$ images obtained by using $n$ different monochromatic radiations $E_{1}, E_{2}, \ldots, E_{n}$, will be examined in the next section, together with determination of the concentration of only a part of the components and a detailed analysis of the precision of the quantification procedure. Section 3 is devoted to the correction procedure to apply for taking into account the fluorescence effects and for dealing with situations in which classical X-ray sources being used, the incident photon beams are not strictly monochromatic. At last, some aspects of bremsstrahlung induced AXRM will be briefly indicated in appendix.

\section{The proposed approach.}

2.1 GENERAL CONSIDERATIONS. - For obtaining elemental (or species) mapping, the first principle of analytical X-ray microscopy consists in obtaining various images of a given specimen from the use of some selected incident photon energies. For the experimental point of view, the acquisition of one image can be obtained in a sequential (scanning) mode, like in STEM or directly on a two-dimensional detector like in TEM. But in opposition to electron microscopy, there is next a wide variety of technical solutions which differ from each other by the choice of the X-ray source (from the X-rays generated by the electron bombardment of a target up to the use of the synchrotron radiation), by the choice of the optical arrangement (with focussing elements such as zone plates or without them in contact microscopy or in shadow microscopy) and by the choice of the detectors. Figure 1 illustrates some of these possible arrangements and their resolving power are indicated in the captions.

Using one of these arrangements the principle of the conventional approach (differential X-ray microscopy) for the determination of an element $A$ in a mixture of other elements is illustrated in figure 2. The basic data consist of the measurements of the attenuation of $X$-ray beams having energies $E_{1}$ and $E_{2}$ situated on the two sides of the absorption edge, $E_{\mathrm{A}}$, of the element of interest.

When characteristic radiations are used, the contribution of the matrix is considered like a decreasing background following a power law of the form $\alpha E^{-3}$ [8]. When a tunable X-ray source such as synchrotron radiation is used, it is possible to reduce the $E_{1}, E_{2}$ interval ( $E_{1}=$ $\left.E_{\mathrm{A}}-\varepsilon ; E_{2}=E_{\mathrm{A}}+\varepsilon\right)$ in order to consider that this background contribution is the same for the two measurements [9][13]. In the two cases, the local weight per unit area, $m_{\mathrm{A}}$, is next deduced so that the contrast of the final image $\left(m_{\mathrm{A}}\right.$ image) is also influenced by the possible local change of the thickness of the specimen. More, if the specimen is composed of $n$ elements or chemical species, $2 n$ initial images have in general to be acquired. One may observe that, in principle, only $n$ measurements are necessary to find $n$ unknown values and that the use of a sum rule for the 


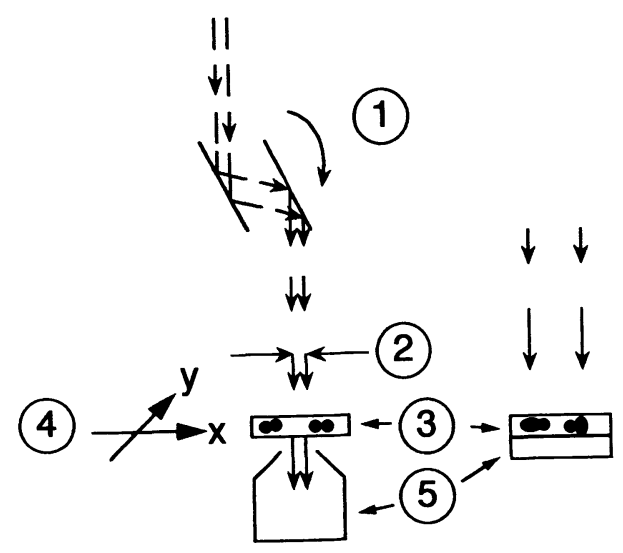

(a1)

(2).

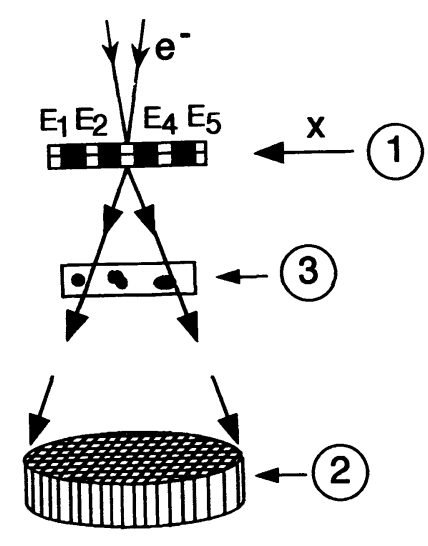

(b)

Fig. 1. - Some possible arrangement for analytical X-ray micr('scopy by using tunable: a) or sequential: b) X-ray sources. The use of synchrotron radiation a) requires the use of a monochromator (1) and either mechanical scanning (4) of the specimen (3) in Fig. a1.- or the use of a removable detector (5) or photoelectronic converter, in Fig. a2.- Element 3 represents the specimen in all the figures. In the scanning mode (a1), the lateral resolution was first governed by the size of the aperture (2) [10]. It has been greatly improved by inserting a focussing element -such as a zone plate- between the aperture (2) and the specimen (3): a $55 \mathrm{~nm}$ spatial resolution has been obtained for the $285-590 \mathrm{eV}$ interval [11][12]. In contact microscopy (in Fig. a2), the use of photoelectronic converters -instead of photo resists- permits to obtain on line digital images, with a lateral resolution of $\cong 0.3 \mu \mathrm{m}$ [13-15]. Derived from the old shadow microscopy but using, now, modern detectors, Fig. $b$ illustrates the arrangement used in our laboratory $[16,17]$ with a CCD camera as a two dimensional detector (2) leading to a lateral resolution in the micron range. The simple shift of the target holder (1) allows to generate successively the characteristic lines $E_{1}, E_{2}, \ldots E_{5}$ issued from different targets set side by side. 


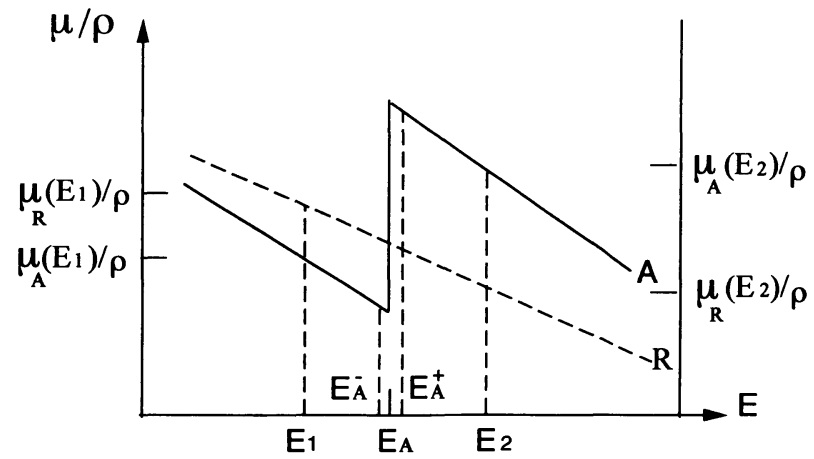

Fig. 2. - Principle of elementary analysis by differential $\mathrm{X}$-ray microscopy. The weight per unit area, $m_{\mathrm{A}}$, (g.cm ${ }^{-2}$ ) of element $\mathrm{A}$, is deduced from the absorption measurements at photon energies $E_{1}$ and $E_{2}$ on the two sides of its absorption edge $E_{\mathrm{A}}$. The use of a tunable $X$-ray source permits to reduce this energetic interval and, thus, to improve the precision on $m_{\mathrm{A}}$ by a better evaluation of the contribution of other elements (dashed line $R$ ).

atomic concentrations $\left(\sum_{K} C_{K}=1\right)$ may permit, in addition, to obtain the local thickness. It is the leading philosophy of the approach described in the following sub-section.

2.2 PROPOSED APPROACH: DETERMINATION OF THE CONCENTRATIONS OF ALL THE COMPONENTS. - In this sub-section, it is assumed that different images of a given specimen have been obtained at different incident photon energies and the goal is to deduce the atomic concentrations $C_{\mathrm{A}}, C_{\mathrm{B}} \ldots, C_{\mathrm{N}}$ of the various components $\mathrm{A}, \mathrm{B}, \ldots \mathrm{N}$ of this specimen from the measured intensities associated with each pixel of the specimen (except specific indications, the following developments apply to any kind of analytical X-ray microscopies and are independent of the lateral resolution of the instrument being operated).

The basic data consist of the measurements of the attenuation of X-ray beams of different photon energies passed through the specimen. Each of the successive radiations are supposed (in this sub-section) to be monochromatic radiations of energy $E_{1}, E_{2}, \ldots, E_{n}$ and their associated incident intensities are represented by the symbols $I_{1}^{0}, I_{2}^{0}, \ldots I_{n}^{0}$ while the symbols $I_{1}, I_{2}, \ldots I_{n}$ represent the transmitted intensities. Each of the incident and transmitted intensities are measured and are associated to a given pixel in the image plane of dimensions $D_{x}, D_{y}$. The symbols $(p, q)$ indicating the position of the pixel of interest will be omitted for the sake of the simplicity because the procedure to apply is the same for all the pixels. Issued from the source, the transmitted Xrays, associated to a given pixel, define an elemental analyzed volume in the object plane. The approximate dimensions of this volume are $\left(D_{x} / M\right) \times\left(D_{y} / M\right) \times t$ where $M$ is the magnification of the X-ray microscope and $t$ the local thickness of the specimen (at point $p, q$ ). The number of atoms contained in such a volume is $n(p, q)=N^{0} t \cdot D_{x} D_{y} / M^{2}$ where $N^{0}$ is the atomic density per unit volume (in the $10^{22} \mathrm{~cm}^{-3}$ range for solids).

In this subsection and like in differential microscopy, the $n$ elements $\mathrm{A}, \mathrm{B}, \ldots \mathrm{N}$, that are supposed to compose the specimen are all known $a$ priori (or postulated) and their unknown corresponding atomic concentrations -to find- are $C_{\mathrm{A}}, C_{\mathrm{B}}, \ldots C_{\mathrm{N}}$ (in at/at).

Using the well-known Beer's law $\left(I=I^{0} \mathrm{e}^{-\mu t}\right)$ and with the help of the expression of the linear absorption coefficient, $\mu$, as a function of the photoabsorption cross-sections $Q_{\mathrm{A}}(E), Q_{\mathrm{B}}(E)$ [i.e. 
$\left.\mu=N^{0} \sum C_{j} Q_{j}\right]$ the following expression is easily obtained for the radiation $E_{1}$ :

$$
i_{1}=\mu_{1} t=\ln \left(I_{1}^{0} / I_{1}\right)=N^{0} t\left[C_{\mathrm{A}} Q_{\mathrm{A}}\left(E_{1}\right)+C_{\mathrm{B}} Q_{\mathrm{B}}\left(E_{1}\right)+\ldots+C_{\mathrm{N}} Q_{\mathrm{N}}\left(E_{1}\right)\right]
$$

For the radiation $E_{j}$ one obtains:

$$
i_{j}=\ln \left(I_{j}^{0} / I_{j}\right)=N^{0} t\left[C_{\mathrm{A}} Q_{\mathrm{A}}\left(E_{j}\right)+C_{\mathrm{B}} Q_{\mathrm{B}}\left(E_{j}\right)+\ldots+C_{\mathrm{N}} Q_{\mathrm{N}}\left(E_{j}\right)\right]
$$

Consequently a system of $n$ linear equations is obtained for each pixel of the image plane. It associates the known values $i_{1}, i_{2}, i_{n}$ (deduced from a logarithmic compression of the measurements) and the quantities $C_{\mathrm{A}}, C_{\mathrm{B}}, \ldots, C_{\mathrm{N}}$ to be determined via the $Q\left(E_{j}\right)$ values which are obtained from tables [18, 19], calculated [20,21], or measured using standards. In classical mathematics text books, this system is known as Cramer's system and is easily solved by evaluating determinants of the $n^{\text {th }}$ order:

$$
C_{K}=\left|D_{K}\right| / N^{0} t \cdot\left|Q\left(E_{j}\right)\right|
$$

where $\left|Q\left(E_{j}\right)\right|$ is the determinant of the cross-sections:

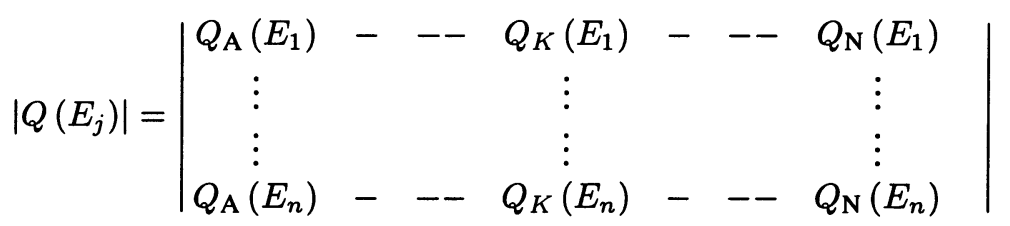

and $\left|D_{K}\right|$ is the determinant deduced from $\left|Q\left(E_{j}\right)\right|$ by substituting the terms of the $K^{\text {th }}$ column: $Q_{K}\left(E_{1}\right), Q_{K}\left(E_{2}\right), \ldots, Q_{K}\left(E_{n}\right)$ by $i_{1}, i_{2}, \ldots i_{n}$ respect:

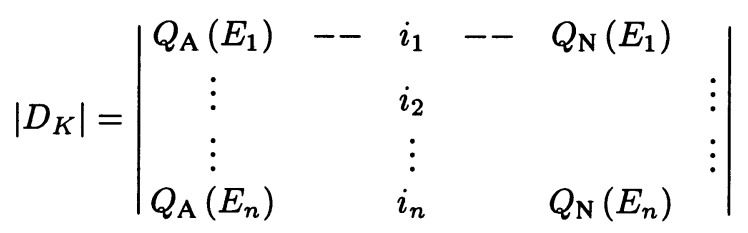

The last unknown value, $N^{0} t$ (surface atomic density, in $\mathrm{cm}^{-2}$ for the pixel under consideration), is next evaluated by writing the sum rule condition:

$$
C_{\mathrm{A}}+C_{\mathrm{B}}+\ldots+C_{\mathrm{N}}=1(\text { or } 100 \%)
$$

For example, for a binary compound, $\mathrm{AB}$, the following results are obtained using 2 monochromatic radiations $E_{1}$ and $E_{2}$ :

$$
\begin{aligned}
& C_{\mathrm{A}}=\left[i_{1} Q_{\mathrm{B}}\left(E_{2}\right)-i_{2} Q_{\mathrm{B}}\left(E_{1}\right)\right] /|Q| N^{0} t \\
& C_{\mathrm{B}}=\left[i_{2} Q_{\mathrm{A}}\left(E_{1}\right)-i_{1} Q_{\mathrm{A}}\left(E_{2}\right)\right] /|Q| N^{0} t
\end{aligned}
$$

with

$$
|Q|=Q_{\mathrm{A}}\left(E_{1}\right) Q_{\mathrm{B}}\left(E_{2}\right)-Q_{\mathrm{A}}\left(E_{2}\right) Q_{\mathrm{B}}\left(E_{1}\right)
$$

and

$$
N^{0} t=\left\{i_{1}\left[Q_{\mathrm{A}}\left(E_{2}\right)+Q_{\mathrm{B}}\left(E_{2}\right)\right]-i_{2}\left[Q_{\mathrm{A}}\left(E_{1}\right)+Q_{\mathrm{B}}\left(E_{1}\right)\right]\right\} \cdot|Q|^{-1}
$$


The results may also be expressed in terms of mass concentrations, $C_{K}^{m}(\mathrm{~g} / \mathrm{g})$ or in terms of weights per unit area $m_{K}\left(\mathrm{~g} \mathrm{~cm}^{-2}\right)$ :

$$
C_{K}^{m}=\left(C_{K} A_{\mathrm{K}}\right) / \sum_{K} C_{K} A_{\mathrm{K}}
$$

and

$$
m_{K}=C_{K}^{m} \rho t
$$

( $A_{\mathrm{K}}$ : atomic mass of element $\mathrm{K} ; \rho$ : local density of the specimen).

The proposed approach also applies to the determination of the concentrations of atoms or molecular compounds (of the form $M_{x} N_{y}$ ) embedded in matrices of well defined chemical composition of the form $P_{z} R_{v}$ (with $x, y, z, v$ whole numbers). For this purpose, in expression (1) and (2), one has only to replace the atomic cross-sections, $Q_{\mathrm{A}}$ and $Q_{\mathrm{B}}$ by the molecular one, $Q_{\mathrm{MN}}$ and $Q_{\mathrm{PR}}$, and the local atomic density $N^{0}$ by the total molecular density for obtaining $C_{\mathrm{MN}}$ and $C_{\mathrm{PR}}$.

2.3 PRECISION ON THE CONCENTRATIONS AND CHOICE OF THE PHOTON'ENERGIES. - From the mathematical point of view, the proposed approach is strictly exact within the validity of the Beer's law (monochromatic radiations) and it admits solutions except when the $\left|Q\left(E_{j}\right)\right|$ determinant is null. It has been tested on simulated images and the initial compositions are exactly deduced from the intensities'values when the same cross-sections values are chosen for the direct and the reverse problems.

From the experimental point of view, the precision, $\Delta C_{\mathrm{K}}$, on the determination of the $C_{\mathrm{K}}$ values is given by:

$$
\frac{\Delta C_{K}}{C_{K}}=\frac{\Delta\left|D_{K}\right|}{\left|D_{K}\right|}-\frac{\Delta\left|Q\left(E_{j}\right)\right|}{\left|Q\left(E_{j}\right)\right|}
$$

This precision depends upon the precision on the cross-sections values and upon the accuracy on the measurements:

i) The precision related to the second term on the right hand side of expression (13) is optimized when the $\left|Q\left(E_{j}\right)\right|$ determinant is maximized. In expression (4), each row of this determinant can be associated to one vector $\mathbf{Q}\left(\mathbf{E}_{1}\right), \ldots, \mathbf{Q}\left(\mathbf{E}_{n}\right)$, . built from its projections $Q_{\mathrm{A}}\left(E_{1}\right), Q_{\mathrm{B}}\left(E_{1}\right)$, $Q_{\mathrm{N}}\left(E_{1}\right)$ on the $n$ orthogonal $Q_{\mathrm{A}}(E), Q_{\mathrm{B}}(E), \ldots, Q_{\mathrm{N}}(E)$ axes. Using the cross-sections tables, it is possible to built various vectors corresponding to various trying photon energies in order to select, next, the energies which optimize the $\left|Q\left(E_{j}\right)\right|$ determinant because they maximize the surface (2 elements), volume ( 3 elements) or hypervolume ( $n>3$ elements) built from the $\mathbf{Q}(\mathbf{E})$ vectors. Figure 3 illustrates such a choice in the case of a binary system.

This choice often corresponds to the choice of energies situated after the threshold energy of each component but there are situations where another choice is better.

If the $a$ priori choice of the photon energies is done by looking at the cross-sections tables, the $Q^{\prime}$ s values derived from measurements on standards have to be used for the final evaluation of the concentrations in order to minimize the $\Delta\left|Q\left(E_{j}\right)\right|$ uncertainty in expression (13).

ii) The precision related to the first term on the right hand side of expression (13) depends upon the precision on the $Q^{\prime}$ s values but also on the precision of the measurements, via the $\Delta i_{j}$ uncertainties:

$$
\Delta i_{j}=n_{o j}^{-1 / 2}\left[1+\exp \mu\left(E_{j}\right) t / 2\right]
$$




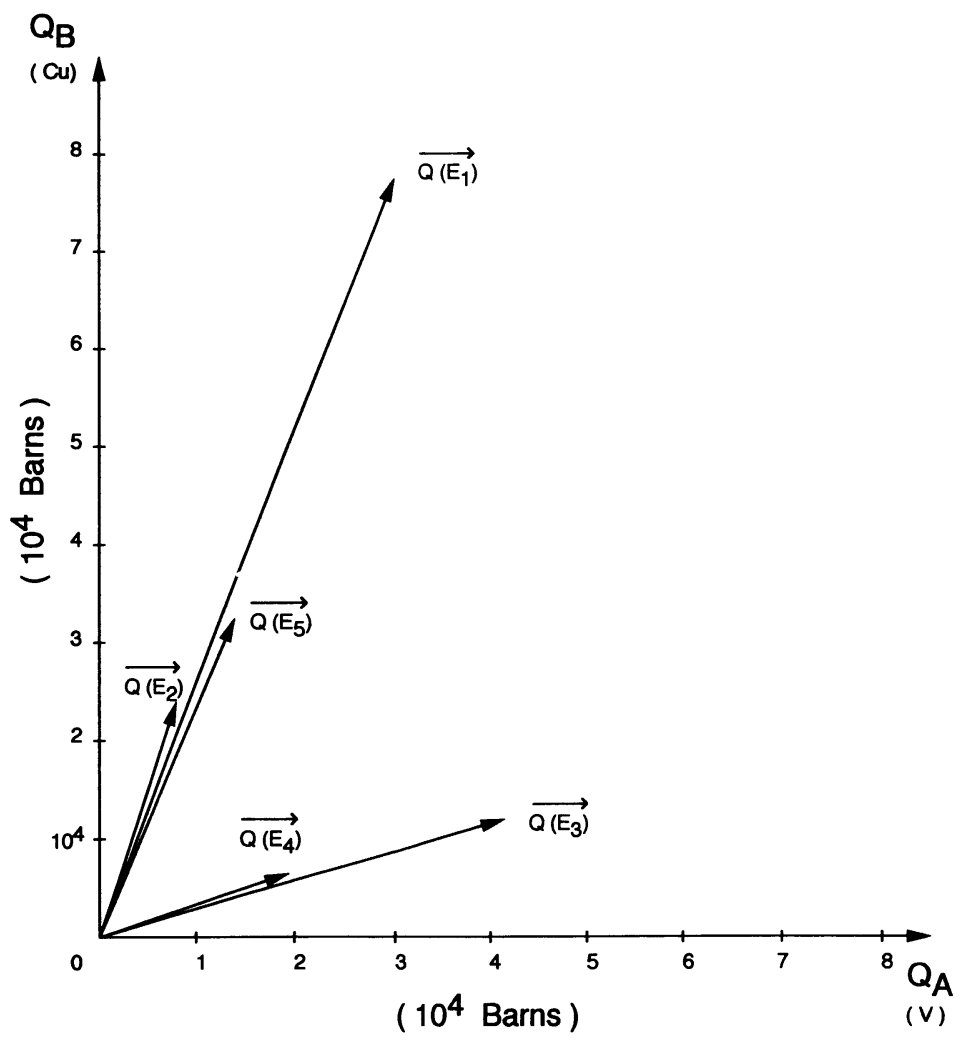

Fig. 3. - For finding the best operating energies (those optimizing the value of the determinant of the crosssections), each possible energy, $E_{j}$, is associated to a vector $\mathbf{Q}\left(\mathbf{E}_{j}\right)$ of components $Q_{\mathrm{A}}\left(E_{j}\right), Q_{\mathrm{B}}\left(E_{j}\right) \ldots$ The best choice corresponds to the maximum surface $(n=2)$ or volume $(n=3)$ built from these vectors and it often corresponds to energies situated after the threshold energies of components. For the binary system shown here $(\mathrm{A}=$ vanadium, $\mathrm{K}$ edge at $5.46 \mathrm{keV} ; \mathrm{B}=$ copper, $\mathrm{K}$ edge at $8.9 \mathrm{keV})$ and for energies ranging from $E_{1}=3 \mathrm{keV}$ up to $E_{5}=9 \mathrm{keV}\left(E_{2}=5 \mathrm{keV} ; E_{3}=6 \mathrm{keV} ; E_{4}=8 \mathrm{keV}\right)$ this best choice is not $E_{3}$ and $E_{5}$ but $E_{1}$ and $E_{3}$ because of the very large $Q^{\prime}$ s values at low energies. The $Q^{\prime}$ s values are taken from reference [18].

(with $n_{o j}$ : number of incident photons for the pixel and the radiation of interest considering only a standard deviation and postulating the same time of acquisition for the incident and the transmitted intensity).

If the uncertainty on the incident intensity is neglected (by increasing the time of acquisition for this intensity) the relative uncertainty on the $i_{j}$ values corresponds to:

$$
\frac{\Delta i_{j}}{i_{j}}=\frac{1}{\sqrt{n_{o j}}}\left(\frac{\mathrm{e}^{x_{j}}}{2 x_{j}}\right) \text { with } x_{j}=\frac{\mu\left(E_{j}\right) t}{2}
$$

From the corresponding curve (see Fig. 4) it is clear that the optimum sensitivity can only be obtained by use of photon energies for which $x$ is close to the unity. Unfortunately it is not possible to take benefit of such a remark for the choice of the photons energies if the approximate composition and thickness of the specimen is not known a priori (to evaluate $\mu \mathrm{t}$ ). 


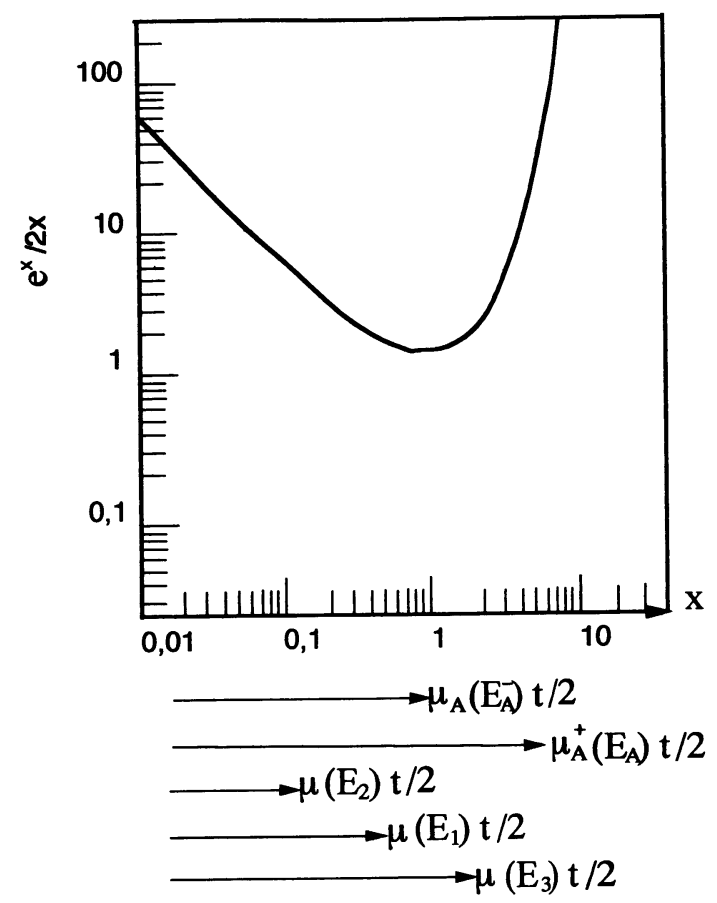

Fig. 4. - The precision on the measurements and the minimum detectable concentration are governed by the function $\mathrm{e}^{x} / 2 x$ with $x=\mu t / 2$. The optimum sensitivity corresponds to $\mu(E) t=2$. Unfortunately, the best energies to operate $E_{1}, E_{2}, E_{3}$ can only be determined with an a priori knowledge of the thickness and composition of the specimen (and they change from pixel to pixel when the local composition or thickness changes). Even in the differential approach the best choice for $E_{\mathrm{A}}^{-}$(before a threshold) may lead to a catastrophic deterioration of the $S / N$ ratio for $E_{\mathrm{A}}^{+}$because a slight change of $\mathrm{E}$ induce a large change on $\mu \mathrm{t}$ (after the threshold of a majority component).

The same causes of uncertainty applies also in the conventional (differential) approach where the precision decreases when the number of independent measurements increases $(2 n$ instead of $n$ ). In addition when the element $A$ of interest is a majority component of the specimen, the slight change of the photon energy from one side to the other side of the absorption edge, $E_{\mathrm{A}}$, leads to a large change of the $\mu \mathrm{t}($ or $x)$ value and a dramatic deterioration of the signal-to-noise ratio is possible.

For the proposed approach, it is suggested to chose the photon energies in order to optimize the $\left|Q\left(E_{j}\right)\right|$ determinant (point $i$, above) when the approximate composition and thickness of the specimen are not known $a$ priori but keeping in mind that this choice can be relaxed when these additional informations are known.

Figure 5 illustrates this strategy. Over the differential approach, one additional advantage of this approach is that it permits the concentration determination of all the components of the specimen even if one of them has a threshold energy outside the investigated energetic interval $\left|E_{\min }, E_{\max }\right|$-element $\mathrm{C}$ of figure 5-. In favourable situations (binary or ternary systems) a precision in the $1 \%$ magnitude is expected for the relative concentration determination. With a poorer precision, the concentration determination of 2 (or more) components can also be performed by using radiations outside the interval of the threshold energies of these components if the corresponding $Q_{\mathrm{A}}(E), Q_{\mathrm{B}}(E)$ curves are not parallel to each other (If the $\mathbf{Q}$ vectors are co- 
linear the Cramer's system does not admit solution). This possibility may be useful for (and has to be restricted to) the investigation of thick specimens that cannot be thinned (living specimen for instance) and which require the use of harder radiations to be investigated. Finally, one may observe that the mathematical procedure to follow for obtaining quantitative elemental mapping is the same for each pixel of the initial images and it is simple enough to perform with a P.C. Using a digital shadow microscope (Fig. 1b), this approach has been applied on test specimens in our laboratory. By changing the target without moving the specimen, successive $\mu\left(E_{j}\right) t-$ or $i_{j}$ images are obtained in few seconds from a logarithmic compression for the acquired images [17]. Few additional seconds are only needed to go from the initial images towards the elemental and thickness maps (paper to be submitted). It has been found that the unique difficulty was related to the non-monochromaticity of the photon beams we use, see section 3.2.

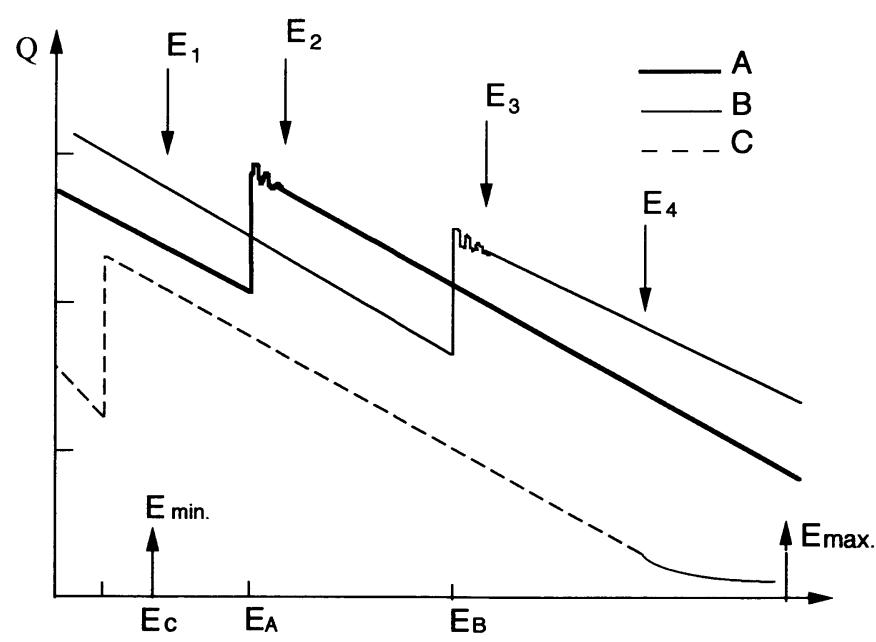

Fig. 5. - For the ternary compound ABC shown here, the best choice for the photons energies nearly corresponds to energies situated just after the threshold energies of each component but slightly higher energies can be chosen to avoid errors associated to the difference of the cross sections of the specimen and the standards associated to near edge structures (see $\left.E_{1}, E_{2}, E_{3}\right) . C_{\mathrm{A}}, C_{\mathrm{B}}, C_{\mathrm{C}}$ can be determinated even if the threshold energy of one component is outside the investigated interval [ $\left.E_{\min }, E_{\max }\right]$. For a binary compound $\mathrm{AB}$, the best choices are $E_{1}$ and $E_{2}$ or $E_{2}$ and $E_{3}$ but $E_{3}$ and $E_{4}$ is possible (with a power precision) and may be useful for the investigation of absorbing specimens that cannot be thinned (case of living specimens for instance).

2.4 DETERMINATION OF ONLY SOME CONCENTRATIONS. - In some cases, some elements of the specimen cannot be distinguished from each others because their cross-section behaviour is very similar in the investigated energy interval $\left[E_{\min }, E_{\max }\right]$. Their contributions look like a monotonous and decreasing background on which the contributions of the other species present discontinuities. For instance it is the case of biological specimens mainly composed of carbon and oxygen (having $\mathrm{K}$ thresholds in the soft $\mathrm{X}$-ray region) when the required identification is that of the relative concentrations of metallic atoms ( $\mathrm{Al}, \mathrm{In}, \mathrm{Ca}, \mathrm{K})$, which can only be done by their $\mathrm{K}$ threshold energies (being situated in explored photon energy range).

In such a situation, it is possible to determine the concentration of only $n^{\prime}$ species over the $n$ components from $n^{\prime}+1$ or $n^{\prime}+2$ measurements. 
For instance, in the case of the concentrations determination of only two elements, A and B (inside a given pixel), the expression (2) can be written in the form:

$$
i_{j}=N^{0} t\left[C_{\mathrm{A}} Q_{\mathrm{A}}\left(E_{j}\right)+C_{\mathrm{B}} Q_{\mathrm{B}}\left(E_{j}\right)+\sum_{J \neq \mathrm{A}, \mathrm{B}}^{N} C_{J} Q_{J}\left(E_{j}\right)\right]
$$

or in the form:

$$
i_{j}=d_{\mathrm{A}} Q_{\mathrm{A}}\left(E_{j}\right)+d_{\mathrm{B}} Q_{\mathrm{B}}\left(E_{j}\right)+F E_{j}^{-R}
$$

where $d_{\mathrm{A}}, d_{\mathrm{B}}$ represent the atomic density per surface unit of elements $\mathrm{A}$ and $\mathrm{B}$ of interest (i.e. $\left.d_{\mathrm{A}}=C_{\mathrm{A}} N^{0} t\right)$ and the power law represents the contribution of the matrix. Strictly speaking, it is not correct to reduce the sum $(\Sigma)$ which appears in expression (16) to a power law having the form $F E^{-R}$ of expression (17) but it has been postulated that all the possible $R_{j}$ values are approximately the same and $R$ is nearly equal to 3 for photon energies below $40 \mathrm{keV}$ [20]. In addition, the term $F E_{j}^{-R}$ may often be considered as a small correction when AXRM is operated in the 5-30 keV for detecting minerals embedded in light matrices (carbon, oxygen or nitrogen) because the terms of the sum decrease like $\sim E_{j}^{-3}$ when $E_{j}$ is increased (i.e. from 1 to $10^{-3}$ when $E_{j}$ goes from $1 \mathrm{keV}$ to $\left.10 \mathrm{keV}\right)$. The result is that in many situations, the exponent $R$ can be chosen to be $R=3$ and $n^{\prime}+1$ images have to be acquired (at $n^{\prime}+1$ different energies) to determine the $n^{\prime}$ surface atomic densities $d_{\mathrm{A}}, d_{\mathrm{B}}, \ldots, d_{\mathrm{N}}$ of $n^{\prime}$ elements plus the factor $F$.

When $F E^{-R}$ cannot be considered as a small correction term with $R=3, n^{\prime}+2$ independent images have to be acquired (for the determination of $F, R, d_{\mathrm{A}}, d_{\mathrm{B}}, \ldots d_{\mathrm{N}}$, ). For this, several procedures can be used such as "Least Squares Method [22]" or better "the Maximum Likelihood Method" [23], using a starting value of the exponent $R$ close to 3. For each pixel, the relative concentrations of the identified elements can, then, be evaluated from the ratios of the form $d_{\mathrm{A}} / d_{\mathrm{B}}=C_{\mathrm{A}} / C_{\mathrm{B}}$. In this approach (illustrated in Fig. 6a) the local determination of $N^{0} t$ cannot be performed because of the impossibility of using the sum relation: $C_{\mathrm{A}}+C_{\mathrm{B}}+\ldots C_{\mathrm{N}}=100 \%$ and when $n^{\prime}$ is $n^{\prime}=1$ or 2 , it does not differ significantly from that used in differential microscopy.

An alternative approach, more closely related to that developed in EELS $[1,2]$, consists in considering the intensities obtained before the thresholds of the elements of interest $\left(E_{\mathrm{A}}, E_{\mathrm{B}}\right)$ as all contributing to a non characteristic background of the form $F^{\prime} E^{-R^{\prime}}$. Then, two first images (obtained at $E_{1}, E_{2}<E_{\mathrm{A}}, E_{\mathrm{B}}$ ) are used to determine $F^{\prime}$ and $R^{\prime}$ in order to evaluate next (by extrapolation) the characteristic contribution of the elements of interest.

Such an approach is illustrated in figure $6 \mathrm{~b}$, allowing determination of the surface atomic density of the element A (for example) from:

$$
i_{3}=d_{\mathrm{A}} Q_{\mathrm{A} k}\left(E_{3}\right)+F^{\prime} E_{3}^{-R^{\prime}}
$$

where $Q_{\mathrm{A} k}\left(E_{3}\right)$ represents the photo-ionization cross-section of the subshell, $k$, of interest of the element $\mathrm{A}$. The relative concentrations $C_{\mathrm{A}} / C_{\mathrm{B}}$ may, then, be deduced.

As in EELS, this procedure is an extrapolation process based on a mathematical model for the background. The use of unconventional methods related to multivariate statistics and entropy concepts (i.e. factor analysis of correspondence or principal component analysis) should be inspected because they run without any a priori hypothesis for the background and the noise [24, 25]. 


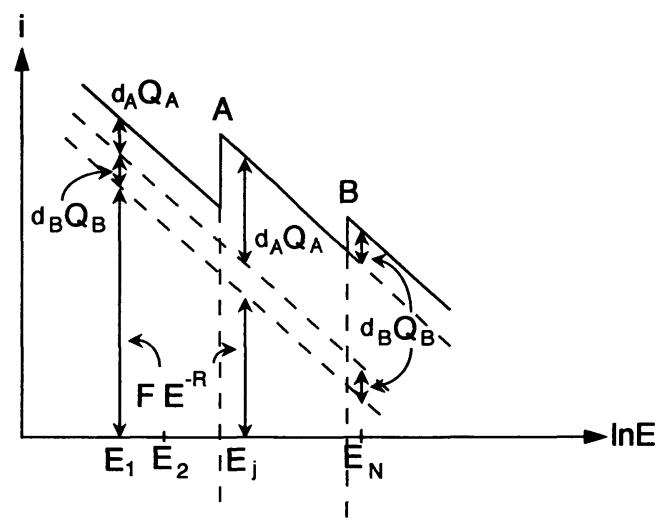

$E_{A} \quad E_{B}$

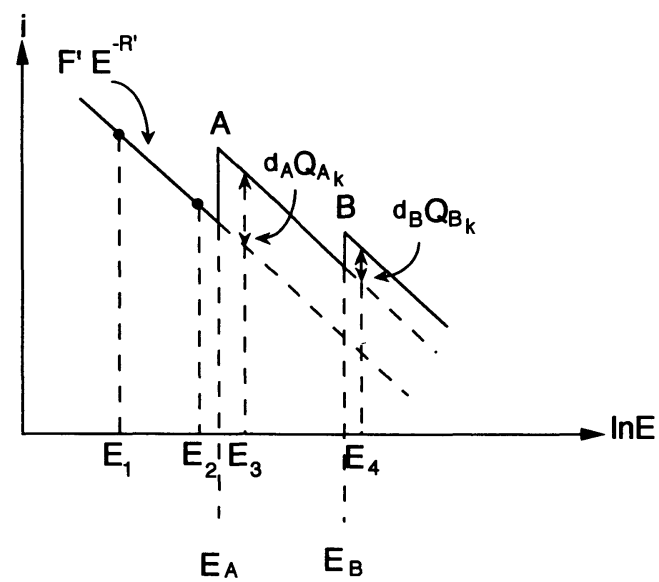

Fig. 6. - Two possible ways for determining the concentration of only some species (here, A and B) embedded in a matrix composed of many unknown elements (or compounds). On the top (a): Illustration of expression (16) showing the contributions to the useful signal of the cross-sections of the elements of interest, $\mathrm{A}$ and $\mathrm{B}$ (on the two sides of their characteristic energies, $E_{\mathrm{A}}$ and $E_{\mathrm{B}}$ ) with also that of the unknown components: $F E^{-R}$. On the bottom (b): the contributions before the threshold 'energies are considered as an unsignificant background following a power law in the form $F^{\prime} E^{-R^{\prime}}$.

2.5 USE OF INTERNAL STANDARDS AND DETECTION LIMITS. - In the approaches described above, all the pixels of a given image are submitted to the same systematic procedure but no correlation between them are used. When some information concerning the concentration values of some given regions of the specimen are previously known, the procedure applied to the other regions can be simplified by comparing the signal intensities obtained in an unknown region to those obtained in the known region.

Such a situation occurs artificially when the investigated specimen and a pure elemental standard are set close together in order to obtain the two parts simultaneously in one image. It is also the case when the investigated specimen is of uniform thickness and is composed of regions of a pure matrix, $M$, beside regions where an elemental impurity, $\mathrm{A}$, is embedded in the same matrix. In that case, the atomic (or molecular) concentration of the impurity, $C_{\mathrm{A}}$ can be deduced from the measurements at two points: $(A+M)$ and $(M)$, of the same single image (obtained with the 
use of only one radiation, $E_{0}$ ).

In such a case, expressions (1) and (2) lead to:

$$
i(\mathrm{~A}+\mathrm{M})=N^{0} t\left[C_{\mathrm{A}} Q_{\mathrm{A}}\left(E_{0}\right)+\left(1-C_{\mathrm{A}}\right) Q_{\mathrm{M}}\left(E_{0}\right)\right]
$$

and

$$
i(\mathrm{M})=N^{0} t \cdot Q_{\mathrm{M}}\left(E_{0}\right)
$$

The final result is:

$$
C_{\mathrm{A}}=\frac{\delta i}{i(\mathrm{M})} \cdot \frac{Q(\mathrm{M})}{\delta Q}
$$

where $\delta i$ is equal to $i(\mathrm{~A}+\mathrm{M})-i(\mathrm{M})$ while $\delta Q$ corresponds to $\left|Q_{\mathrm{A}}-Q_{\mathrm{M}}\right|$.

The result given by expression (21) is only valid when the total surface atomic density, $N^{0} t$, is postulated to be the same for the two investigated regions, $(A)$ and $(A+M)$.

In fact, the main interest of expression (21) is that it allows us to evaluate the minimum atomic concentration detectable, $C_{\mathrm{A}}(\mathrm{min})$ for an element $\mathrm{A}$ (or a compound) embedded in a matrix $\mathrm{M}$. When this expression is combined to expression (15) of $(\Delta i / i)$ and when, as is usually admitted, one considers that a detail in a matrix is detectable when $\Delta n$ is at least equal to 3 times the statistical fluctuations of the photon counting, $n$, besides this detail $(\Delta n \cong 3 \sqrt{n}(\mathrm{M}))$, one obtains:

$$
C_{\mathrm{A}}(\min )=\frac{3 \Delta i}{i} \frac{Q(\mathrm{M})}{\delta Q}
$$

(a 100\% quantum efficiency for photon detectors is assumed).

As it has been previously discussed (Sect. 2.3), the influence of the factor $3 \Delta i / i$ is that the sensitivity of X-ray microscopy as well as that of X-ray tomography [17-26,27] is optimum when the function $\mathrm{e}^{x} / 2 x$ is minimum $(x=\mu \mathrm{t} / 2)$ and this minimum is close to the unity when $\mu t \cong 2$. For an order of magnitude, $3(\Delta i / i)_{\mathrm{MDC}}$ is of around $10^{-2}$ when $n_{0}$ (per pixel) is of around $10^{5}$ photons with the optimum choice of $E: E_{\text {opt }}$. For a specimen of given uniform thickness and composition, this means that this optimum sensitivity can only be obtained by using this optimum photon energy, $E_{\text {opt }}$, (for which $\mu \mathrm{t} \cong 2$ ) and that the other images (obtained at other energies and required for performing AXRM) will have a deteriorated $S / N$ ratio if the magnitude of the number of incoming photons is the same for all the energies. When the specimen is strongly heterogeneous in thickness or composition, only some regions of an image may correspond to the optimization of $S / N$ and the location of these optimized regions changes when the photon energy is changed. At the end of the image process, one may expect to have a more or less uniform $S / N$ in each of the elemental maps obtained finally but these effects are intrinsic effects and are independent from the approach being applied for chemical mapping.

To evaluate the sensitivity in terms of $C_{\mathrm{A}}(\mathrm{min}$ ) (in Exp. (22)), one has also to take into account the factor $Q(\mathrm{M}) / \delta Q$ which strongly influences the final result. As expected, one finds that an element $\mathrm{A}$ cannot be detected when its cross-section $Q_{\mathrm{A}}$ is very close to that of the matrix, $Q_{\mathrm{M}}$, in which it is embedded. Cracks or empty cavities in the specimen correspond to $Q_{\mathrm{A}}=0$ and the sensitivity in their detection is governed by the factor $\Delta i / i$ (Exp. (15)) only. A similar situation occurs when low absorbing elements $\mathrm{A}$ are in highly absorbing matrices, $\mathrm{M}$ (i.e. when $Q_{\mathrm{A}} \ll Q_{\mathrm{M}}$ ).

On the other hand, there are favourable situations for which this factor, $Q(\mathrm{M}) / \delta Q$, is in the $2 \times 10^{-2}-10^{-3}$ interval. From the tabulated values of $Q$ [18], it can be seen for instance that this situation occurs for A elements such as $\mathrm{Si}(Z=14)$ up to $\mathrm{Zn}(Z=30)$, when they are embedded in a carbon matrix and are investigated at $E>E_{\mathrm{A}}(K)$. At the same photon energies 


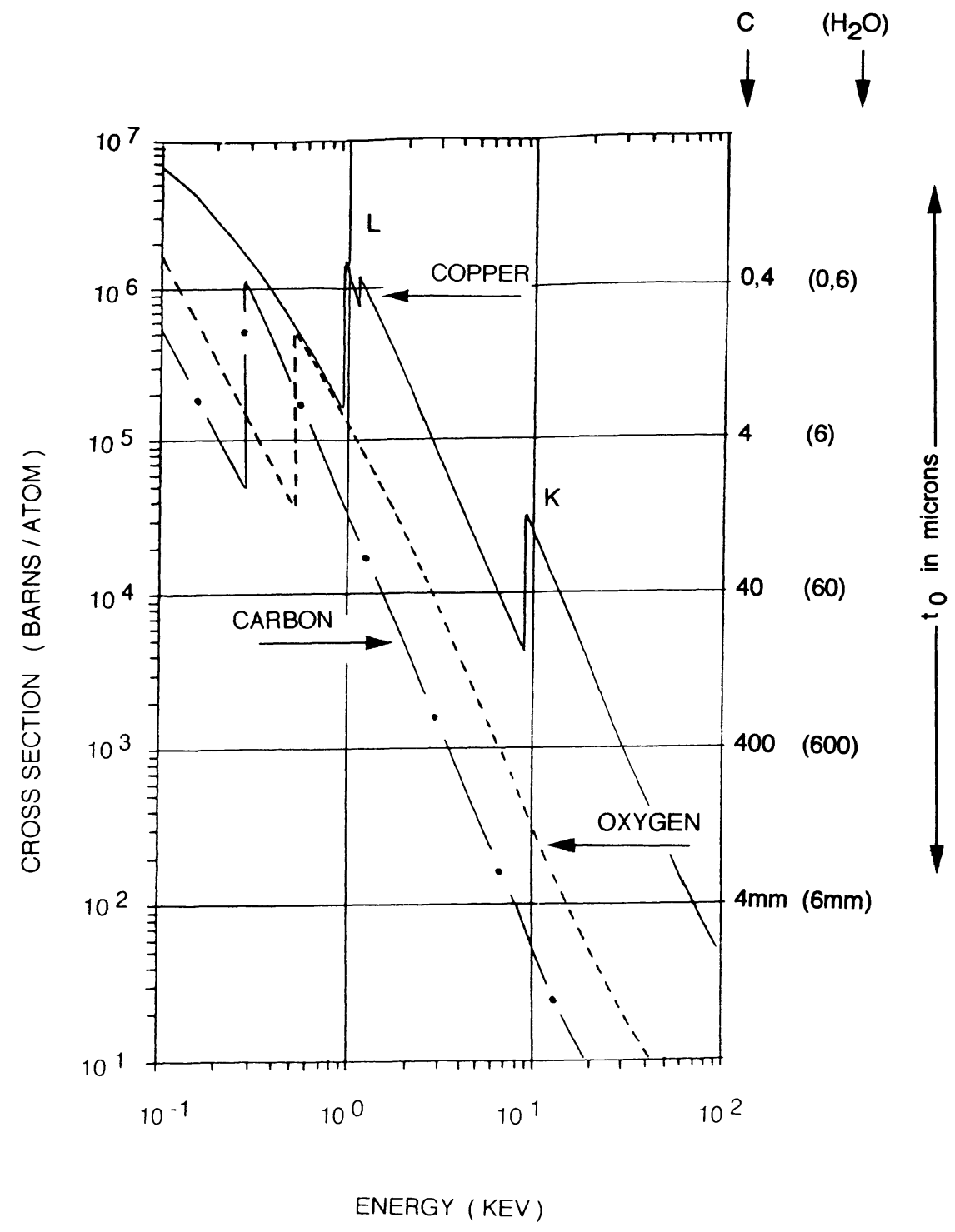

Fig. 7. - When $\mathrm{Cu}$ impurities are embedded into carbon or water matrices, $\mathrm{M}$, the detection limit $C_{\mathrm{Cu}}$ (min), given by expression (22), may reach the $10^{-4}-10^{-5}$ interval for photon energies larger than $E_{\mathrm{Cu}}(\mathrm{K}) \cong$ $8.9 \mathrm{keV}$ because $Q(\mathrm{M}) / \delta Q$ is $\cong 10^{-2}-10^{-3}$. These results holds for matrices having the optimum thickness $t_{0}$ (vertical scale on the right) and for $\cong 10^{5}$ incident photons leading to $3 \Delta i / i \cong 10^{-2}$. The $Q(E)$ curves are deduced from reference [18]. The situation is less favourable for photon energies situated in the water window, between the $\mathrm{C}$ and the $\mathrm{O} \mathrm{K}$ edges at $0.28 \mathrm{keV}$ and $0.53 \mathrm{keV}$, and the optimum thickness is far thinner.

and if oxygen (of water) is chosen as a matrix (instead of carbon), the factor $Q(\mathrm{M}) / \delta Q$ is in the $5 \times 10^{-2}-5 \times 10^{-3}$ interval for the same A elements. Figure 7 illustrates this point for copper at energies larger than $E_{\mathrm{Cu}}(K) \cong 8.9 \mathrm{keV}$. Consequently, one specific advantage of X-ray microscopy operated at such energies is that its sensitivity may reach $10^{-4}-10^{-5}$ (atomic fraction) for medium 
$Z$ elements embedded in very light matrices of carbon or water such as those of biological tissues. It is interesting to point out that such results are better than those expected when the selected photon energies are inside the so-called "water window" [28] and if such high sensitivities may only be obtained on specimens having the optimized thickness, $t_{0}=2 / \mu$, this thickness is far larger than that needed for the investigation in the soft energy range (see the vertical scale on the right of Fig. 7).

To summarize this sub-section one has to outline that when compared with many other analytical techniques, the sensitivity of X-ray microscopy covers a larger range from its inability to detect some elements in some matrices $(\Delta C>1)$ up to its excellent detection limits for some other elements in other matrices. This is because the value of this sensitivity depends not only upon the statistics of countings but also on the very large change of the cross-sections of elements of interest and of the matrix as well as on the exponential influence of the thickness of the specimen.

\section{Correction procedures.}

In the relations (1), (2) and others, it has been admitted that the incoming radiations $I_{1}^{0}, I_{j}^{0}$, are monochromatic radiations and that the collected intensities are directly related to the absorption process (given by the Beer's law).

In fact, the photoabsorption process (on which analytical X-ray microscopies are based) is followed by a radiative de-excitation process which leads to the emission of a fluorescence intensity, $I_{\mathrm{F}}$ (basic mechanism of X-ray fluorescence analysis technique, XRF).

The recorded intensities, $I_{\mathrm{R}}$, are the sum of the transmitted intensities $I_{j}$ and sometimes of the fluorescent intensities, $I_{\mathrm{F}}$ and it is not possible to separate the two contributions when nonanalyzer detectors (such as CCD cameras) are used.

On the other hand, when conventional X-ray sources are used to produce the incoming radiations (electron bombardment of targets in laboratory X-ray microscopes), these incoming radiations are not monochromatic because of the bremsstrahlung contribution. Here again, these two contributions to the recorded intensities, $I_{\mathrm{R}}$, cannot be discriminated except when analyzers (such as EDS) are interposed between the source and the detector. The correction procedures to apply for taking into account these effects are the subject of the following sub-sections.

3.1 FLUORESCENCE CORRECTION. - Where a specimen is irradiated by a parallel incident beam of photons having an energy $E_{0}$ the transmitted intensity decreases as $I=I^{0} \mathrm{e}^{-\mu\left(E_{0}\right) t}$, while the number of fluorescent photons of energy $E_{\mathrm{F}}^{\mathrm{A}}$ and generated by atoms $\mathrm{A}$ in the specimen increases with the path of the X-rays into the specimen.

These fluorescent photons are emitted isotropically and are, in turn, partly attenuated with a linear absorption coefficient $\mu\left(E_{\mathrm{F}}^{\mathrm{A}}\right)$. Qualitatively one may expect that the fluorescence effect, $I_{\mathrm{F}}$, can be neglected when the exponent $\mu\left(E_{0}\right) t$ is below few unities but its weight (with respect to $I$ ) is expected to increase above $I$, when almost all the incident photons are attenuated. In the inspection of the final X-ray image, the fluorescence effect may become significant for strongly absorbing volumes. For an estimate, if it is assumed that half of the fluorescent photons are going in the forward direction and are parallel to the transmitted beam, the following expression can easily be obtained $[29,30]$ :

$$
\frac{I_{\mathrm{F}}\left(E_{\mathrm{F}}^{\mathrm{A}}\right)}{I^{0}\left(E_{0}\right)}=\frac{1}{2}\left[N^{0} C_{\mathrm{A}} Q_{\mathrm{A} k} \omega_{\mathrm{K}}(\mathrm{A})\right] \frac{\mathrm{e}^{-\mu\left(E_{\mathrm{F}}^{\mathrm{A}}\right) t}-\mathrm{e}^{-\mu\left(E_{0}\right) t}}{\mu\left(E_{0}\right)-\mu\left(E_{\mathrm{F}}^{\mathrm{A}}\right)}
$$

where $\omega_{K}(\mathbf{A})$ is the fluorescence yield of element $\mathbf{A}$. 


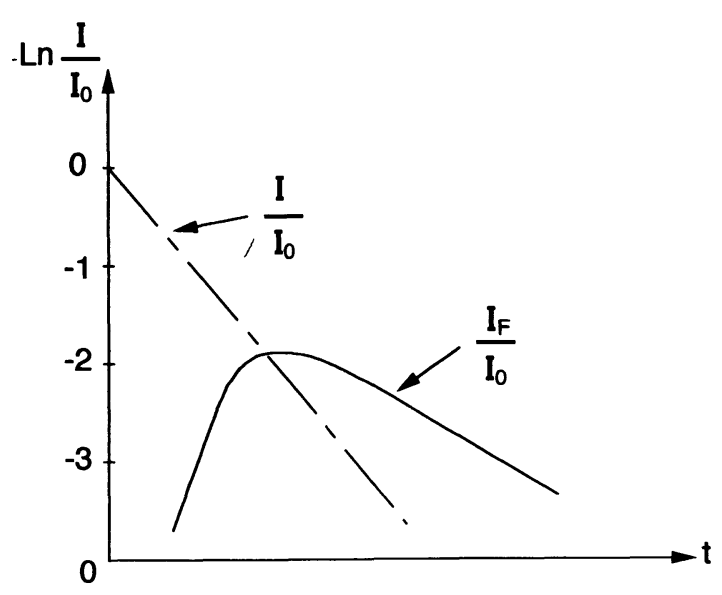

Fig. 8. - Approximate change as a function of $t$ (thickness of the specimen) of the transmitted intensity $I$ (dotted line) and of the generated fluorescent intensity $I_{\mathrm{F}}$ (full line). Vertical scale in arbitrary (ln) units for the two curves (from Exp. (23)).

Figure 8 shows the approximate behaviour of $I_{\mathrm{F}}$ as a function of the thickness of the specimen and this behaviour is compared to the corresponding change of the transmitted intensity, $I\left(E_{0}\right)$.

The weight of the corresponding fluorescence correction depends upon the specific experimental arrangement being used. It can be neglected in the scanning mode or when the specimen is not in direct view of the detector. But it may be a significant correction when the specimen is widely illuminated and when the fluorescent or the elastically scattered photons may reach this detector.

Fluorescence correction is also a matrix correction which depends upon the composition $C_{\mathrm{A}}$, $C_{\mathrm{B}}, \ldots C_{\mathrm{j}}$ of the specimen so that the final evaluation has to include the sum of all the fluorescent contributions as well as the elastic diffuse contributions and the response function of the detector $r\left(E_{\mathrm{F}}^{\mathrm{A}}\right), r\left(E_{\mathrm{F}}^{\mathrm{B}}\right), \ldots, r\left(E_{\mathrm{F}}^{j}\right), r\left(E_{0}\right)$ for the various radiations it receives.

If sophisticated calculations for $I_{\mathrm{F}}$ can be performed, the key point is that the weight of such fluorescence corrections depends upon the type of X-ray microscope being used. As illustrated in figure $9 \mathrm{a}$, in contact microscopy, the fluorescent intensity received by a given pixel in the image plane is issued only from its corresponding region in the object plane and from the neighbouring regions. On the other hand, when the detector is far from the object (as in shadow microscopy), all the irradiated parts of the specimen (even those which are outside the field of the image) may contribute to the more or less uniform background due to fluorescent signals (Fig. 9b).

In the case of contact microscopy, an iterative procedure can be used for the fluorescent correction. In a first step, the measured intensities for a given pixel are used to evaluate $C_{\mathrm{A}}^{\prime}, C_{\mathrm{B}}^{\prime}, C_{j}^{\prime}$ without corrections. Next the $I_{\mathrm{F}}$ contributions (issued from the corresponding region and its proximity in the object plane) are evaluated by using expressions similar to expression (23) in order to be substracted from the measured intensities. Using the intensities thus corrected, the operations are then repeated.

Again, for widely illuminated specimens in direct view of the detector when the distance between the specimen and the detector is large, the fluorescence correction is expected to be greater than in contact microscopy but it is easier to estimate experimentally because it is quite uniform for all the pixels. For instance a $\mathrm{Si}(\mathrm{Li})$ detector (see Fig. 9c) can be set between the specimen and the two-dimensional detector in order to measure the relative weight of the fluorescent contributions with respect to the transmitted intensity and the same corrections are next applied to all 


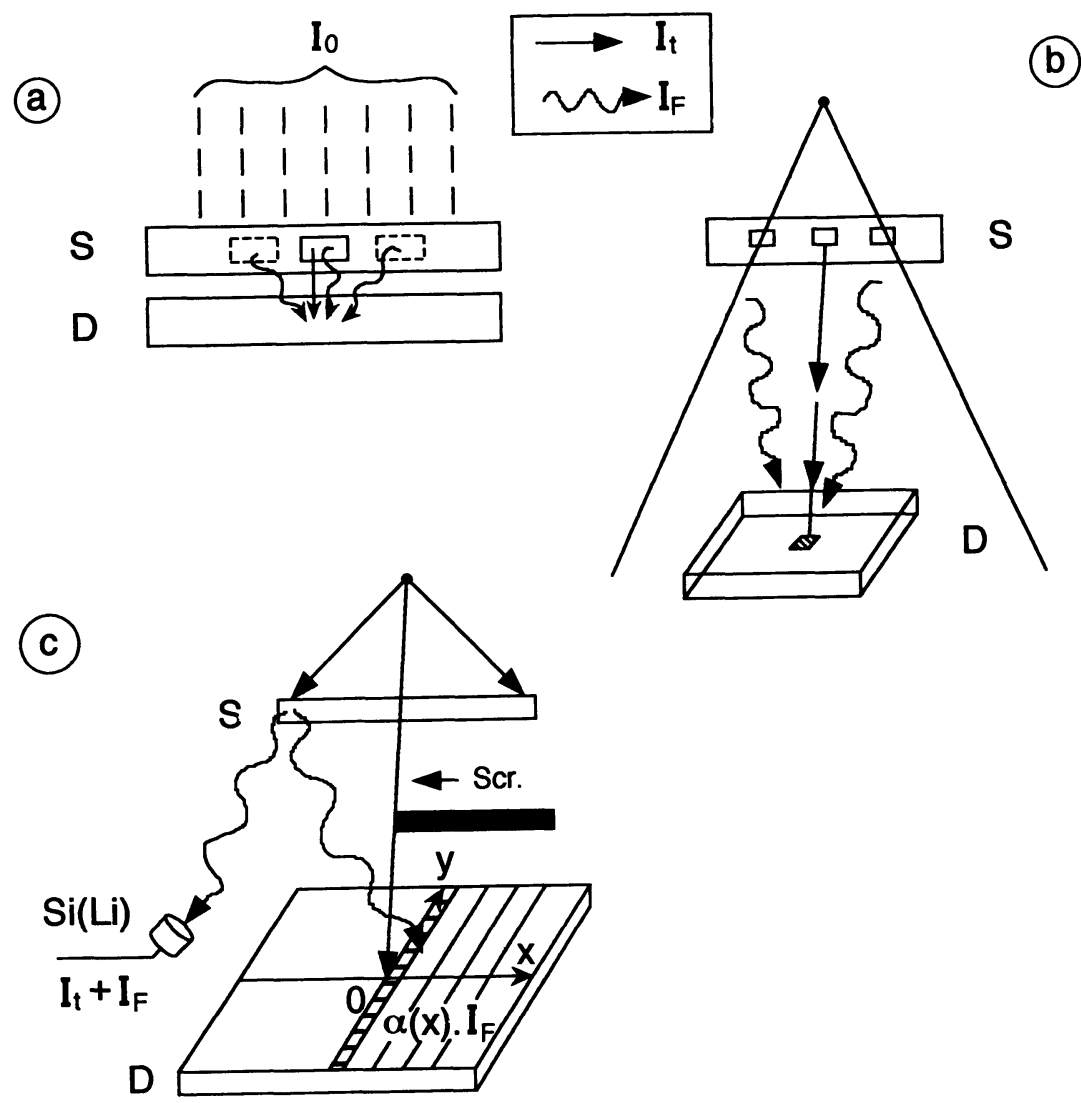

Fig. 9. - The fluorescent contribution may be significant when the specimen is widely illuminated and is in direct of the detector: contact microscopy (a) and shadow microscopy (b). Possible solutions for evaluating experimentally the weight of the fluorescent contributions (c) in shadow microscopy by either using a $\mathrm{Si}(\mathrm{Li})$ detector or, better, by inserting a knife edge screen. The intensity received by each pixel situated along the border ( $y$ direction) in the shadow region corresponds to the fluorescent intensity emitted by the left hand part of the specimen. The contribution emitted by the righthand part of the specimen can be measured in the same way by setting the blade on the left hand side. To perform fluorescence correction, the sum of these mean intensities (per pixel) is next subtracted from the intensity received by each pixel of the initial image. The same knife-edge method may also sign the possible diffraction effects in a crystalline specimen by Kossel patterns in the shadow region.

pixels of a given image. A better alternative solution consists in inserting a half screen (like a knife edge) between the specimen and the two-dimensional detector in order to obtain two equivalent areas (on the screen). The area being in the shadow region contains only a part of the fluorescent (and of the elastically diffused) contribution but no signal intensity. This contribution is not uniform; it is maximum along the border of the two regions where it corresponds to the fluorescent contribution of the half of the specimen which is on the opposite side of the shadow.

Consequently the proposed method for the fluorescence correction consists in measuring the mean intensity received by each pixel along this border for one position of the knife edge and next in performing the same operation when the knife edge is set in a symmetrical position. Finally the sum of the mean intensities received by the pixels (being along the border and successively in the shadow region) is subtracted from the intensity received by each pixel of the initial image. 
The correction can be improved by verifying that the mean fluorescent intensity received by each pixel along the border is nearly a constant. The advantage of this procedure (as compared to the use of a $\mathrm{Si}(\mathrm{Li})$ detector) is that it does not require knowledge of the response function of the detector as a function of the photon energy and it can be easily applied to the fluorescent correction of non-monochromatic illuminations. Finally, one may observe that the knife edge method may also indicate the occurrence of diffraction effects in shadow microscopy of crystalline materials by the appearance of pseudo-Kossel lines in the shadow region.

3.2 NON-MONOCHROMATIC INCIDENT IRRADIATIONS. - As mentioned at the beginning of this section, the subject of this sub-section concerns the use of non strictly monochromatic irradiations such as those issued from a metallic target submitted to the bombardment of an electron beam, and one may either deal with this non monochromaticity by finding a mathematical procedure (i) or by reducing it using a suitable experimental arrangement (ii).

i) Mathematical procedure:

In this procedure, the spectral distribution of the generated $\mathrm{X}$-ray photons is considered to be composed of one main monochromatic part (incoming intensity: $I_{j}^{0}$, photon energy: $E_{j}$ ) and of a continuous part. The monochromatic part may include several characteristic lines of the same family such as $\mathrm{K} \alpha 1, \mathrm{~K} \alpha 2, \mathrm{~K} \beta 1$ if their weight (as $100 \%, 50 \%, 15 \%$ ) is taken account for the evaluation of their mean cross-section. The continuous part may include some other characteristic lines and is described by its spectral distribution $g_{j}(E)$. When the specimen is off the beam, the recorded intensity (related to a given pixel) is now:

$$
I_{j}^{0}(\mathrm{R})=r\left(E_{j}\right) \cdot I_{j}^{0}+\int_{0}^{E_{\mathrm{p}}} r(E) \cdot g_{j}(E) \mathrm{d} E
$$

where $r(E)$ again represents the response function of the detector and $E_{\mathrm{p}}$ is the maximum photon energy in the spectral distribution (it is the energy of the primary electron beam when the X-rays are generated by electron bombardment).

When the specimen is irradiated by the X-ray beam, the intensity measured on the same pixel is given by:

$$
I_{j}(\mathbf{R})=r\left(E_{j}\right) I_{j}^{0} \mathrm{e}^{-\mu_{j} t}+\int_{0}^{E_{\mathrm{p}}} r(E) \cdot g_{j}(E) \mathrm{e}^{-\mu(E) t} \mathrm{~d} E
$$

The exponent $\mu(E) t$, in the integral, corresponds to the function:

$$
\mu(E) t=N^{0} t\left[C_{\mathrm{A}} Q_{\mathrm{A}}(E)+C_{\mathrm{B}} Q_{\mathrm{B}}(E)+\ldots C_{\mathrm{N}} Q_{\mathrm{N}}(E)\right]
$$

Out of the integral, the exponent $\mu_{j} t$ is the specific value of the above function at the energy $E_{j}$ and it is directly related to the quantity $i_{j}$ developed in expression (2).

In order to correlate the present situation to that of the monochromatic situation described in sub-section 2.2, we need to know the response function of the detector $r(E)$ (which was not required in the monochromatic case) and the spectral distribution $g(E)$. The latter may be deduced from experiments using a solid-state detector (as in Fig. 9) or from the use of more or less refined theoretical calculations like that widely explored for the background modeling in electron probe microanalysis, see for instance reference [3], chapter 8, p.393. The knowledge of $r(E)$ and $g(E)$ allows us to calculate (analytically or numerically) the integral appearing in expression (24) in order to deduce the quantity $r\left(E_{j}\right) I_{j}^{0}$ from the measured intensity $I_{j}^{0}(\mathrm{R})$ and this quantity can also be put into the first term in the right hand side of expression (25).

If the number of elements composing the specimen is $n, n$ different pairs of measurements similar to $I_{j}^{0}(\mathrm{R})$ and $I_{j}(\mathrm{R})$ have to be performed in order to obtain $n$ expressions similar to (24) 
and (25). The first term on the right hand side of each expression represents the monochromatic contribution while the second, the integral, represents the continuous correction but both terms are functions of the required concentrations $C_{\mathrm{A}}, C_{\mathrm{B}}, \ldots C_{\mathrm{N}}$, and of $N^{0} t$. In a first step, it is possible to neglect the continuous correction in order to estimate the approximate values $C_{\mathrm{A}}^{(1)}, C_{\mathrm{B}}^{(1)} \ldots, C_{\mathrm{N}}^{(1)}$ for the concentrations and $\left(N^{0} t\right)^{(1)}$ for $N^{0} t$. This step is exactly that which has been described in sub-section 2.2 for the concentration determination in the monochromatic situation. It is then possible to insert these approximate values into expression (26) in order to evaluate the continuous correction (to the first order) by calculating the integral in the right hand side of expression (25).

The results of such integrations being $S_{1}^{(1)}, S_{2}^{(1)}, \ldots, S_{j}^{(1)}, S_{n}^{(1)}$, it is then easy to substract these results from the corresponding recorded intensities $I_{1}(\mathrm{R}), I_{2}(\mathrm{R}), I_{j}(\mathrm{R}), I_{n}(\mathrm{R})$ in order to find more realistic concentrations and $N^{0} t$ values $\left(\right.$ i.e. : $\left.C_{\mathrm{A}}^{(2)}, C_{\mathrm{B}}^{(2)}, \ldots, C_{\mathrm{N}}^{(2)}\right)$ from expressions having the following form:

$$
\ln \left[\frac{r\left(E_{j}\right) \cdot I_{j}^{0}}{I_{j}(\mathrm{R})-S_{j}^{(1)}}\right]=N_{0} t^{(2)}\left[C_{\mathrm{A}}^{(2)} Q_{\mathrm{A}}\left(E_{j}\right)+C_{\mathrm{B}}^{(2)} Q_{\mathrm{B}}\left(E_{j}\right)+\ldots+C_{\mathrm{N}}^{(2)} Q_{\mathrm{N}}\left(E_{j}\right)\right]
$$

The next step of this iterative process is to evaluate the second order correction $S_{1}^{(2)}, S_{2}^{(2)}, \ldots$, $S_{j}^{(2)}, S_{n}^{(2)}$ from the $C_{\mathrm{A}}^{(2)}, C_{\mathrm{B}}^{(2)}, \ldots, C_{\mathrm{N}}^{(2)}$ values, and so on.

The process has to be stopped at the correction of the kth order when the difference between the $C^{(k)}$ and $C^{(k+1)}$ values falls into the interval of uncertainty, $\Delta C / C$ of the order of magnitude of $\Delta i / i$ as given by expression (24) and situated in the $1 \%$ range.

The detailed calculations of the successive corrections $S^{(1)}, S^{(2)} \ldots$ require the use of numerical values $[18,19]$ or analytical expressions $[20,21]$ for the change of the cross-sections of the elements of interest as a function of $E$.

In practice, the key difficulty of the iterative method is that it has to be applied to each pixel because the beam hardening is a matrix effect which may vary from one point of the object to another one. To win computation time, another method can be applied to specimens composed of a reduced number of elements or well defined compounds.

For a binary compound for instance, the number of values to find is only two, $C_{\mathrm{A}}$ and $N^{0} t,\left(C_{\mathrm{B}}\right.$ being deduced from the sum rule). It is then possible, from expression(25) to calculate $a$ priori all the expected intensities $I_{j}(\mathrm{R})$ when $C_{\mathrm{A}}$ is changing from $0 \%$ to $100 \%$ and $t$ is changing from saying few microns up to few tens of microns. A reference scale of the calculated intensities is then established to which the experimental intensities obtained for each pixel can be compared. For evaluating the integral in expression (25) the use of a data base giving the $Q_{\mathrm{A}}(E)$ and $Q_{\mathrm{B}}(E)$ values is necessary.

But before performing any kind of mathematical correction, the following points have to be kept in mind:

- In the incident radiation, the relative weight of the characteristic line, $I_{j}^{0}$, with respect to the continuous distribution, $\int_{0}^{E_{\mathrm{p}}} g_{j}(E) \mathrm{d} E$, depends on the nature of the elemental target and on the $E_{\mathrm{p}}$ value. It can be estimated to range from 10 or more (light elements, low $E_{\mathrm{p}}$ values) down to $\mathrm{O} .1$ (in the less favourable cases).

- The spectral distribution of the transmitted beams depends on the nature of the investigated specimen and its thickness.

- The proposed procedure is based on a subtraction and the correcting term can be much greater than the final corrected term so that the question of the final accuracy must be asked, first.

It is clear that the suggested procedure is mainly useful for improving the results obtained in favourable cases where the characteristic part of the transmitted signal remains larger (or at least 
has the same order of magnitude) than the continuous part of this signal. This constraint implies that the thickness of the specimen is less or at least equal to the optimum thickness, $t_{0}=2 / \mu$, for characteristic radiation $\left(\mathrm{e}^{-2}=0.135\right)$. Given this constraint, these corrections provide an original way to reduce the beam hardening artefacts well known in X-ray radiography and X-ray tomography [31, 32].

ii) Experimental procedure:

One of the ways to isolate a specified line from a non-monochromatic beam is by use of filters. Among various possible arrangements, the use of a pair of balanced filters seems very promising for AXRM based on conventional X-ray sources. The principle is known from a long time (Ross, 1928 [33]) and has been commonly used in fluorescence studies [34] or diffraction studies [35]. A recent paper also suggest their use in X-ray cone-beam microtomography [36].

A balanced filter consists of two separately mounted thin foils of elements with absorption edges lying just above, $E_{R_{2}}$ and just below, $E_{R_{1}}$, the energy desired. In shadow microscopy a first image can be obtained by setting one of the two filters between the thin foil target and the camera (for instance below the thin foil target and in contact to it). A second image is next obtained wich an identical thin foil target and the second filter. The difference, pixel by pixel of these two images will give an image corresponding to the use of a radiation limited in energy in the pass band between the two absorption edges $E_{R_{1}}$ and $E_{R_{2}}$.

This can be accomplished by the adjustment of the thickness of the two filters in order to i) obtain the exact balance between the two filters for the radiations outside the pass band and ii) optimize the useful signal intensity into the pass band.

The first point is achieved when the ratio, $k$, of the filters thicknesses obeys to:

$$
k=t_{R_{1}} / t_{R_{2}}=\mu_{R_{2}}^{0} / \mu_{R_{2}}^{0}
$$

where the subscripts $R_{1}$ and $R_{2}$ mean filter 1 and filter 2 respect and $\mu^{0}$ represent their linear absorption coefficient at energies outside the pass band energy $E_{R_{1}}, E_{R_{2}}$.

The second point is achieved by maximazing the difference between the two intensities transmitted in the pass band energies $E_{i}$. Elementary differentiation leads to:

$$
t_{R_{1}}=\left(k \mu_{R_{1}}^{i}-\mu_{R_{2}}^{i}\right)^{-1} \operatorname{Ln}\left(k \mu_{R_{1}}^{i} / \mu_{R_{2}}^{i}\right)
$$

where $\mu_{R_{1}}^{i}, \mu_{R_{2}}^{i}$ are the linear absorption coeff. at energies inside the pass band. For example the successive use of a $5.3 \mu \mathrm{m}$ thick chromium foil and of a $4.6 \mu \mathrm{m}$ thick manganese foil permits a $66 \%$ transmission of the $\mathrm{Fe} \mathrm{K}_{\alpha}$ line intensity at $6.4 \mathrm{keV}$ in the energy interval $\mathrm{Cr} \mathrm{K}$ edge (5.99 $\mathrm{keV}$ ) - Mn Kedge (6.54 keV). It also permits the removal of the $\mathrm{Fe} \mathrm{K}_{\beta}$ contribution -for additional details, see reference [36].

Like for the mathematical procedure described previously, this experimental procedure is based on a subtraction for the signals and here on the addition for the noise of the two initial images. The addition of the noises leads to an increase of the acquisition time for obtaining a final image having the same signal-to-noise ratio as that of images acquired in monochromatic conditions. Also this procedure suffers from the same constraints related to the relative weight of the characteristic contribution $I_{j}^{0}$ with respect to the continuous contribution and to the choice of the thickness of the investigated specimen. A specific advantage is associated to the ease of operation of digital images acquired with CCD cameras.

A specific drawback is due to the fact that the measured intensities may contain a significant fluorescence contribution issued from the filters -mainly the filter $R_{1}$ having an absorption edge below the characteristic energy desired-.

In fact the two methods are not in opposition and it would be interesting to combine them. For instance the mathematical method can be applied to the images deduced from the Ross method 
in order to eliminate the residual contribution of the continuous radiation associated to the nonexact balance between the two filters.

\section{Conclusion.}

In this paper, I have tried to show that the elemental identification of species is possible in Xray microscopy using various incident photon energies and various experimental arrangements. A new procedure leading to the determination of the concentrations of all or only some of the components has been indicated. An expression for the detection limit of AXRM has also been given and the optimizing conditions for the thickness of the specimen and the incident photon energy have been next deduced.

The detected intensities may differ from the theoretically transmitted intensities so that it is necessary for the former, to correct the fluorescence effects and the deviations in the monochromaticity. The corresponding correction procedures have been also suggested and a positive application of the bremsstrahlung (i.e. the bremsstrahlung induced AXRM) is also indicated in appendix. Due to the development of modern detectors having a high sensitivity, a good linearity and a high dynamic range, the rapid acquisition of digital $\mathrm{X}$-ray images is now possible and these images can easily be processed in order to achieve, finally, quantitative elemental maps.

Concerning the key point of this paper, related to a new quantification procedure for elemental mapping by $\mathrm{X}$-ray (absorption) microscopy, the main advantages of the proposed approach (with respect to the conventional approach named differential analysis) are:

i) The minimization of the different radiations to be used whose number is strictly equal to the concentrations to determine.

ii) The possibility to map an element or more having a threshold energy outside the investigated energy interval.

iii) The simultaneous acquisition of thickness maps (more precisely $N^{0} t$ maps) which permit to distinguish between the thickness contrast and the chemical contrast of the initial images.

iv) The minimization of the number of irradiations being used permits to hope an improved signal-to-noise ratio for a given dose received by the specimen or the minimization of the received dose for a given (final) signal-to-noise ratio.

In our laboratory, the corresponding experimental developments in X-ray microscopy are in progress and we hope to submit in the near future a companion article demonstrating the practical use of the concepts developed here. In the longer term, these concepts will also be applied to X-ray microtomography (following the way initiated by Thompson et al. [37] and Hirano et al. [38]) and to dynamic studies in X-ray microscopy [39]. At last, one may observe that some of the concepts developed here may also be adapted to conventional radiography and tomography.

\section{Acknowledgements.}

The author is grateful to his colleagues P. Trebbia, D. Mouze and S. Rondot for friendly and stimulating discussions. This work has been partly supported by INSERM: grant 910903 . 


\section{Appendix: overview of the bremsstrahlung induced AXRM}

Up to now this microscopy has not been developed and its principle is derived partly from recent experiments in computerised tomography (C.T.) for element specific imaging [40] partly from the principle of AXRM with synchrotron sources. It consists in using the high energy tail of the bremsstrahlung generated by electron bombardment at variable primary electron beams energies, $E_{\mathrm{p}}$ in order to obtain various $\mathrm{X}$-ray images of the specimen forprimary energies $E_{\mathrm{p}_{1}}, E_{\mathrm{p}_{2}}, \ldots, E_{\mathrm{p}_{n}}$, in such a way that the $E_{\mathrm{p}_{1}}, E_{\mathrm{p}_{2}}$ interval includes the threshold energies, $E_{\mathrm{T}}^{\mathrm{A}}, E_{\mathrm{T}}^{\mathrm{B}}, \ldots, E_{\mathrm{T}}^{\mathrm{N}}$ of the elements to be mapped.

When $E_{\mathrm{p}}$ crosses a given threshold energy $E_{\mathrm{J}}$ the high energy tail of the bremsstrahlung suffers an extra-absorption effect due to the jump of the absorption cross-section of element $J$. The position in energy where the deficit of transmitted intensities occurs indicates the presence of the element and the amplitude of this deficit, at $E_{\mathrm{p}}>E_{\mathrm{J}}$, is an increasing function of its concentration.

This principe is illustrated in figure 10 for the detection of an element $\mathrm{A}$. For a given pixel, the intensity, $I_{\mathrm{p}_{1}}$, recorded for a primary beam energy $E_{\mathrm{p}_{1}}$, is given by:

$$
I_{\mathrm{p}_{1}}=\int_{0}^{E_{\mathrm{p}_{1}}} g_{\mathrm{T}}\left(E, E_{\mathrm{p}_{1}}\right) \cdot r(E) \mathrm{e}^{-\mu(E) t} \mathrm{~d} E
$$

This form looks like the integral appearing in the right hand side of expression (25), but here, the spectral distribution, $g_{\mathrm{T}}$ includes the contribution of the characteristic lines and it is also a function of the changing primary beam energy $E_{\mathrm{p}}$.

For the same pixel the intensity, $I_{\mathrm{p}_{n}}$ recorded for $E_{\mathrm{p}_{n}}\left(>E_{\mathrm{A}}\right)$ can be written in the form:

$$
I_{\mathrm{p}_{n}}=\int_{0}^{E_{\mathrm{p}_{n}}} g_{\mathrm{T}}\left(E, E_{\mathrm{p}_{n}}\right) \cdot r(E) \mathrm{e}^{-\left[\mu(E)+\Delta\left(\mu^{\prime} E\right)\right] t}
$$

with

$$
\Delta \mu(E) t=N^{0} t . C_{\mathrm{A}} Q_{\mathrm{A}_{k}}(E)=d_{\mathrm{A}} Q_{\mathrm{A}_{k}}(E)
$$

In computerized tomography, Markham and Fryar [40] perform two measurements at energies $E_{\mathrm{p}_{1}}$ and $E_{\mathrm{p}_{2}}$ before the threshold energy $E_{\mathrm{A}}$, to determine the values of the constants to be used to describe the function $\mu(E)$ analytically. The tube activity constant (or the constant which appears in Kramer's relationship for the spectral distribution of the bremsstrahlung) is deduced from a measurement without the specimen. Also starting from, at least, two measurements (below $E_{\mathrm{A}}$ ), an alternative solution consists in extrapolating these results to the energy $E_{\mathrm{p}_{n}}$ (above the threshold) in order to evaluate the intensity that would be obtained in the absence of threshold, $I^{\prime} \mathrm{p}_{n}$, i.e.:

$$
I_{\mathrm{p}_{n}}^{\prime}=\int_{0}^{E_{\mathrm{p}_{n}}} g\left(E, E_{\mathrm{p}_{n}}\right) r(E) \mathrm{e}^{-\mu(E) t} \mathrm{~d} E
$$

the deficit in intensity due to the extra-absorption effect, is:

$$
I_{\mathrm{p}_{n}}^{\prime}-I_{\mathrm{p}_{n}}=\Delta I=\int_{E_{\mathrm{T}}^{\mathrm{A}}}^{E_{\mathrm{p}_{n}}} g_{\mathrm{T}}\left(E, E_{\mathrm{p}_{n}}\right) r(E) \cdot \mathrm{e}^{-\mu(E) t}\left(1-\mathrm{e}^{-\Delta \mu(E) t}\right) \mathrm{d} E
$$

In the restricted interval $E_{\mathrm{A}}, E_{\mathrm{p}_{n}}$, the response function of the detector can be considered as a constant, the Kramer's relationship can be used to describe the useful spectral distribution (the 

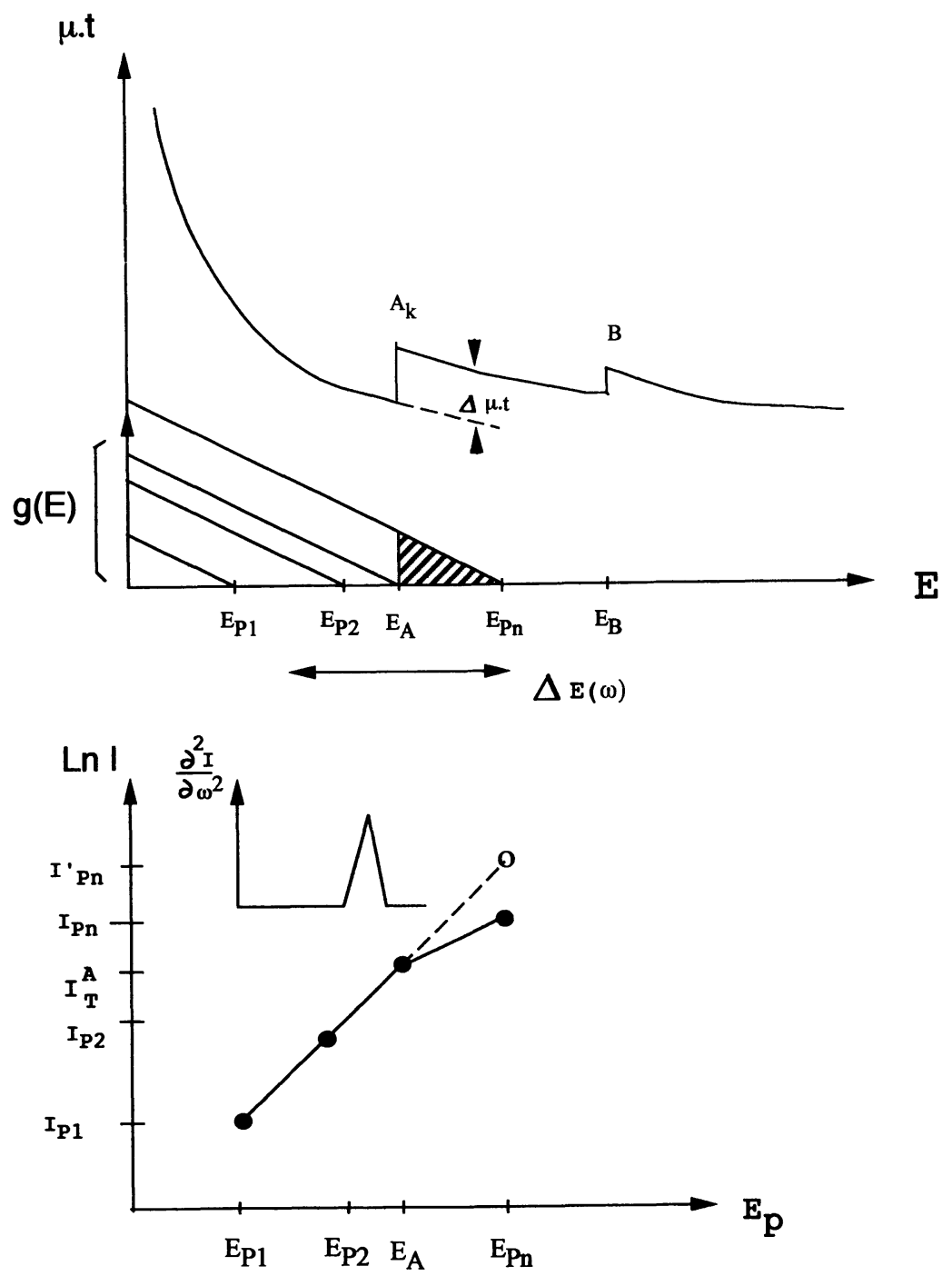

Fig. 10. - Principle of bremsstrahlung -induced- AXRM. Various images are obtained at different primary beam energies $E_{\mathrm{p}_{1}}, E_{\mathrm{p}_{2}}, \ldots E_{\mathrm{p}_{n}}$. The $E_{\mathrm{p}_{1}}-E_{\mathrm{p}_{n}}$ interval crosses the absorption edge energy, $E_{\mathrm{A}}$, of a given element, $\mathrm{A}$. The jump of $\mu \mathrm{t}$ at $E_{\mathrm{A}}$, (top curve of the top figure) induces an extra absorption above $E_{\mathrm{A}}$ in the spectral distribution of the bremsstrahlung (hatched area in the bottom curve of the top figure). From the recorded intensities $I_{\mathrm{p}_{1}}, I_{\mathrm{p}_{2}}, \ldots$ measured at energies $E_{\mathrm{p}} \leq E_{\mathrm{A}}$ the intensity being expected at $E_{\mathrm{p}_{n}}$ (but without extra-absorption) can be deduced by an extrapolation process using preferably a vertical log.scale; this intensity is $I_{\mathrm{p}_{n}}^{\prime}$ (open symbol in the bottom figure). The difference between $I_{\mathrm{p}_{n}}^{\prime}$ and the intensity $I_{\mathrm{p}_{n}}$ (measured for $E_{\mathrm{p}}=E_{\mathrm{p}_{n}}$ ) represents the extra-absorption effect. Elemental mapping can be performed by obtaining first and second difference images the primary beam energy $E_{\mathrm{p}}$ being tuned around $E_{\mathrm{A}}$. The insert illustrates also the possibility of X-ray absorption spectroscopy using a lock-in amplifier in the second derivative mode (analog detection).

characteristic lines must have energies below $\left.E_{\mathrm{p}_{1}}\right)$ and a power law of the form $F^{\prime} E^{-3}$ can be used for the normal change of $\mu(E)$.

In order to evaluate, next, the searched value, $d_{\mathrm{A}}$, it is possible to compare the "measured" $\Delta I$ 
values to a serie of results calculated from the integral (in Exp. (34)) by using a set of " $d_{\mathrm{A}}$ " values chosen inside a reasonable interval. This approach is similar to the second method suggested for the bremsstrahlung correction in section 3.2.

A simplified solution consists in evaluating the most appropriate mean value of $\overline{Q_{\mathrm{A}_{k}}(E)}$ for extracting the factor $\left(1-\mathrm{e}^{\overline{\Delta \mu}(E) t}\right)$ out of the integral in order to find directly $d_{\mathrm{A}}$. This last method is not very different from that applied when wide band-width and tunable X-ray sources are used and the appropriate mean cross-section can be deduced from similar experiments on reference specimens. The choice of $E_{\mathrm{p}_{n}}$ is governed by the compromise between the use of a large $E_{\mathrm{T}}^{\mathrm{A}_{k}}-$ $E_{\mathrm{p}_{n}}$ interval for obtaining a significant difference, $I_{\mathrm{p}_{n}}^{\prime}-I_{\mathrm{p}_{n}}$, and a reasonable accuracy for the determination of $I_{\mathrm{p}_{n}}^{\prime}$ by extrapolation. This compromise leads to estimate the interval to few hundred of $\mathrm{eV}$.

In fact, the precision to be expected for the concentrations will be less than the use of perfectly monochromatic radiations. The sensitivity of the method will be also poorer because the useful signal is the difference between two measurements while the noise is the addition of the noise of the two measurements. It is a situation similar to the choice of the Ross filters of section 3.2 and which can be minimized here, by the use of suitable filters for attenuating the photons of low energies.

For semi-quantitative AXRM, the advantages of the method are:

i) a rapid identification of the elements composing the specimen which have threshold energies inside the operating energies of the incident electron beam (from $\sim 3 \mathrm{keV}$ up to $30 \mathrm{keV}$ in X-ray projection microscopes based on the modifications of a scanning electron microscope).

ii) a rather good energy resolution for such an identification, despite the lack of tunable and monochromatic $\mathrm{X}$-ray sources or X-ray analyzers.

iii) The ease to develop this AXRM in a shadow microscope without changing the nature of the target and by using, for instance, the experimental arrangement of Cheng et al. [41].

iv) Also in shadow microscopy, the improvement of the lateral resolution, $r$, via the decrease of the effective X-ray source size " $s$ " because of the reduced penetration depth of the incident electrons generating the useful high energy photons (see for instance Ref. [16] for the relation between $r$ and $s$ ).

v) In scanning X-ray microscopy as developed in a S.E.M. [42-44] but using an analogue detector (photodiode, proportional counter) the possibility of obtaining first and second difference spectra following the method originally developed by Shuman and Kruit for EELS [45]. It should also be pointed out that a deep modulation of the primary beam energy may be easily obtained by the voltage modulation of the target around the earth potential.

vi) The expected sensitivity of the method for the identification and the detection of medium or heavy elements embedded in biological matrices. In such a case, the exponent, $\mu(E) t$, in expression (34) will act like a small correction to the useful signal (see remark, Sect. 2.4).

For all these positive aspects, it seems obvious that the corresponding experimental verifications have to will be done in the near future.

\section{References}

[1] Colliex C. in Advances in Optical and Electron Microscopy. R. Barer and V.E. Cosslett Eds. (Academic Press London) 9 (1984) 65.

[2] EgERTON R.F. in Electron Energy Loss Spectroscopy in the Electron Microscope (Plenum Press NY 1986) Chap. 3-4. 
[3] GoldSTEIN J.I., NEwBury D.E., Echlin P., Joy D.C., FioRi C., LiFSHIN E. in Scanning Electron Microscopy and X-ray microanalysis (Plenum Press NY, 1981) Chap. 5-6.

[4] Cliff G., LORIMER G.W., J. Microsc. 103 (1975) 203.

[5] Prutton M., BARKShIRE I.R., El Gomati M.M., Greenwood J.C., KenNy P.G., RoberTs R.H., Surf. Interf. Anal. 18 (1992) 295.

[6] Glocker R., Frohmayer W., Ann. Phys. 76 (1925) 369.

[7] ENGSTROM A. in X-ray Microanalysis in Biology and Medicine (Elsevier Publishing Co, 1962) 9.

[8] LindSTROM B. in X-ray Optics and X-ray Microanalysis, M.M. Pattee, V.E. Cosslett and A. Engstrom Eds. (Academic Press. NY, 1963) 13.

[9] BUCKLEY C.J., FoSTER G.F., BURGE R.E., Ali S.Y. and SCOTCHFORD C.A. in X-ray microscopy III, A.G. Michette, G.R. Morrisson and C.J. Buckley Eds. (Springer Verlag, Berlin) Springer Series Opt. Sci. 67 (1992) 423.

[10] HoROWITZ P. and HowELL J.A., Science 178 (1972) 608-611.

[11] Ade H., Zhang X., CAMERon S., Costello S., KirZ J., Williams S., Science 258 (1992) 972.

[12] BuCKLEY C.J., KHALEQUE N., Ali S.Y., SCOTCHFORD C.A., DownES S., Di SAlVo L., Proceed. of the 4th International Conf. on X-ray microscopy (Chernogolovka, Russia, Sept.20-24. 1993) To appear in X-ray microscopy IV.

[13] Bigler E. and PolaCK F., J. Phys. Suppl. C 45 (1984) 83.

[14] POLACK F., Thesis University of Orsay (1991) unpublished. POLACK F., LowENTHAL S., PHILIPPOU D. and FouRNET P. in $X$-ray microsc. II, 41, Sayre D., Howells M., Kirz J., Rarback H., Eds. (Springer Verlag, Berlin) Springer Series Opt. Sci. 56 (1987) 220.

[15] KinOShITA K., MATSUMURA T., INAGAKI Y., HIRAI N. in X-ray microsc. III, Springer Series Opt. Sci. 67 (1992) 335.

[16] Thomas X., CazauX J., Erre D., Mouze D., Collard P. in X-ray microsc. III. A.G. Michette, G.R. Morrisson and C.J. Buckley Eds. (Springer Verlag, Berlin, Heidelberg) Springer Series Opt. Sci. 67 (1992) 190.

[17] Erre D., Mouze D., Thomas X., CaZauX J., Inst. Phys. Conf. Series 130 (1993) 567.

[18] VEIGELE W.M.J., X-ray cross-section compilation. Kaman Science Corp. Colorado Springs, Ca 80907 Report 24113 F (1971).

[19] Henke B.L., Lee P., TANAKa T.J., Shimabukuro R.L. and FujiKaWa B.K., At. Data Nucl. Data Tables 27 (1982) 1.

[20] THINH T.P. and LEROUX J., X-ray Spectrom. 8 (1979) 85.

[21] HEINRICH K.F.J. in Proceedings of the 11th International Congress on X-ray optics and Microanalysis, J.D. Brown and R.H. Packwood Eds. (1986) 67.

[22] Bevington P.R. in Data Reduction and error analysis for the Physical Sciences, P.R. Bevington Ed. (Mc Graw Hill, NY, 1969).

[23] TrebBIA P., in Quantitative Microbeam Analysis, NATO ASI Series, E. Fitzgerald Ed. (40th Scottish Summer School in Physics, Dundee, 1992) -to appear.

[24] TreBBiA P. and BonNET N., Ultramicroscopy 34 (1990) 165.

[25] TREBBIA P. and MORY C., Ultramicroscopy 34 (1990) 179.

[26] GrodZINS L., Nucl. Instr. Methods 206 (1983) 541.

[27] Flannery B.P. and Roberge W.G., J. Appl. Phys. 62 (1987) 4668.

[28] KIRZ J. and RARBACK H., Rev. Sci. Instr. 56 (1985) 1.

[29] SPARKS C.J., Adv. X-ray Anal. 19 (1976) 19.

[30] Cazaux J., J. Phys. Lett. 40 (1979) L. 227.

[31] RuSs J.C. in Computer - Assisted Microscopy, J.C. Russ Eds. (Plenum Press, New York and London) Chap. 13 (1990) 419.

[32] BARRETT H.H. and SwINDELL W. in Radiological Imaging. H.H. Barrett and W. Swindell Eds. (Academic Press, NY London) 2 (1981) 438.

[33] Ross P.A., J. Opt. Soc. Am. 16 (1928) 433.

[34] KIRKPATRICK P.K., Rev. Sci. Instrum. 15 (1944) 223.

[35] GUINIER A., Theorie et Technique de la radiocristallographie (3ème Edition Dunod Paris, 1964) Chap. 1,3 , p. 20.

[36] HAM Y.S., RuSS J.C., GARDNER R.P., VergheSE K., Appl. Radiat. Isot. 44 (1993) 1313.

[37] ThOMPSON A.C., Llacer J., FinMan L.C., Hugues E.B., OtIS J.N., Wilson S. and ZeMAN H.D., Nuclear Instrum. Methods Phys. Res. 222 (1984) 319.

[38] Hirano T., EguChi S. and Usami K., Jpn. J. Appl. Phys. 28 (1989) 135. 
[39] Rondot S., Cazaux J., Erre D., Mouze D., Trebbia P., Proceedings of the 3rd EUROMAT (European Conference on Advanced Materials) Paris, June, 1993. To appear in Journal de Physique C.

[40] MARKHAM C. and FrYAR J., Nuclear Instrum. Methods Phys. Res. A 324 (1993) 383.

[41] Cheng P.C., ShinozaKi D.M., Lin T.H., NEWBerRy S.P., SRIDHAR R., TARNG W., CHEN M.T., ChEN L.H. in X-ray microsc. III. Springer Series Opt. Sci. 67 (1992) 184.

[42] MouZe D., CAZAuX J., ThOMAS X., Ultramicroscopy 17 (1985) 269.

[43] El Khaldi A., CaZauX J., Mouze D., Thomas X., in X-ray microsc. III, A.G. Michette, G.R. Morrisson and C.J. Buckley Eds. Springer Series Opt. Sci. 67 (1992) 427.

[44] MouZE D., Doctorate Thesis (University of Reims, February, 1991), unpublished

[45] SHUMAN H. and KRUIT P., Rev. Sci. Instrum. 56 (1985) 231. 